\title{
Plant Layout and Simulation Roaming System based on Virtual Reality Technology
}

\author{
Liang Dong ${ }^{1}$, Zhifeng Wang ${ }^{1}$ \\ ${ }^{1}$ Shanghai Second Polytechnic University, Institute of intelligent manufacturing and control engineering, Shanghai 201209, china
}

\begin{abstract}
The layout and roaming of machinery and equipment in the workshop is an important development direction of intelligent manufacturing. In this paper, the general process of developing an intelligent factory system are put forward. The 3D models of various kinds of mechanical equipment are established. The textures and maps of the model are processed. The virtual simulation engine is used to establish the workshop map. The roaming work of the virtual workshop is developed based on the map. The space layout of the mechanical equipment and the machine tool simulation cutting system are developed. Comparing with the traditional simulation software, this system takes shorter period of development and achieves better visualization. And combined with the virtual reality technology makes this system more creative.
\end{abstract}

\section{Introduction}

Virtual reality (VR) is a kind of computer simulation system that can create the virtual world and let people experience it. It uses computer to generate simulation environment. It is a system simulation with multi-source information fusion, interactive three-dimensional dynamic view and entity behaviour [1-3]. Recent years, numerous research achievements of introducing VR technology into the field of intelligent plant construction have been made. Yu Qiang and others studied the layout of factory processes in three-dimensional interactive environment [4]. Wen Huaixing and others designed a virtual assembly system of parallel machine tools based on Virtools [5]. Liang Hongbao discussed threedimensional scene modelling technology and 3D scene optimization technology [6]. Zhu Wenhua developed the engineering training system of reducer and virtual reality combination [7].

In general, Numbers of domestic scholars have studied the field of virtual roaming technology. This article takes the mechanical engineering training workshop of Shanghai Second Polytechnic University as an example to develop the scene roaming of the workshop practical training machinery.

\section{System framework}

First of all, the 3D model of all kinds of mechanical equipment is established. And the image processing makes the effect closer to the reality. The virtual workshop roaming system is developed under the Unity3D software. Finally, the virtual reality equipment zSpace is applied to realize the lathe simulation system with VR and AR functions. The specific development process is shown in Figure 1.

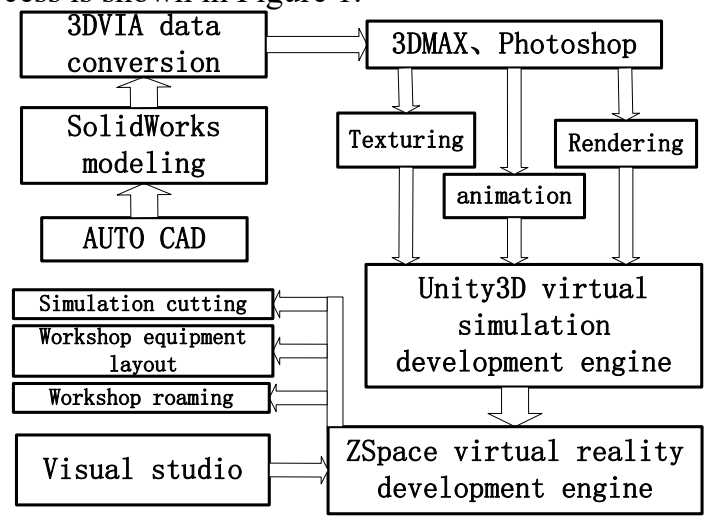

Figure 1. System framework.

\section{Virtual factory model establishment}

At the beginning of the system development, the first step is to do the survey and statistics of the actual workshops, especially the number and placement of all kinds of mechanical equipment in the workshop. table 1 is the number and names of all kinds of mechanical equipment.

Table 1. Factory equipment statistics

\begin{tabular}{|c|c|c|c|c|}
\hline $\begin{array}{c}\text { Device } \\
\text { name }\end{array}$ & $\begin{array}{c}\text { Ordinary } \\
\text { lathe } / \\
\text { table }\end{array}$ & $\begin{array}{c}\text { Ordinary } \\
\text { milling } \\
\text { machine } \\
\text { / table }\end{array}$ & $\begin{array}{c}\text { Numerical } \\
\text { control } \\
\text { lathe/ } \\
\text { table }\end{array}$ & $\begin{array}{c}\text { Outer } \\
\text { grinding } \\
\text { machine } \\
\text { / table }\end{array}$ \\
\hline Number & 12 & 5 & 10 & 2 \\
\hline
\end{tabular}




\subsection{Establishment of equipment model}

Since Unity3D software itself does not have modelling engineering, SolidWorks software is used to establish a three-dimensional model of all kinds of equipment (Figure 2). All of the equipment size parameters are measured by actual measurement, which ensures the reliability of the model.

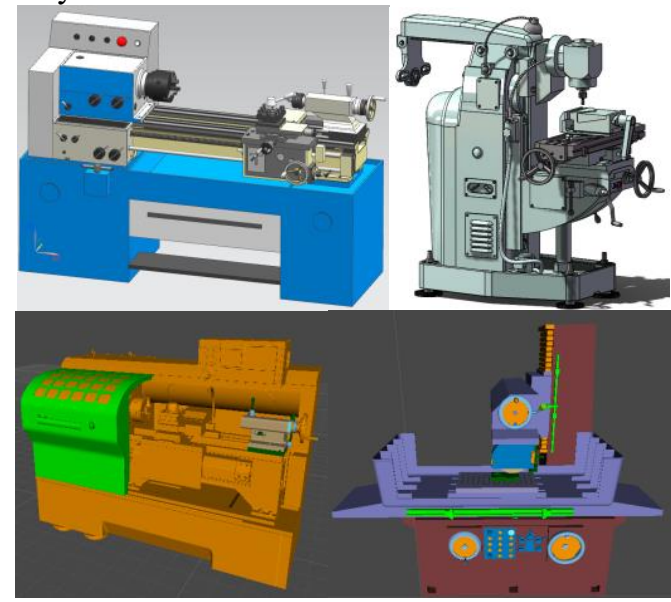

Figure 2. 3D model of mechanical equipment

\subsection{Add texture and map}

In order to make the virtual model closer to the actual model, the texture mapping operation of the model is carried out in detail. First, the surface pattern of the actual machine is photographed by the camera. The image is introduced into the Photoshop software for the correction processing as the material. The mapping operation is carried out in the SketchUp. Fig. 3 is a comparison of the map processing effect of the front and rear panels of ordinary lathe operation. It is obviously that the processed model is closer to the real effect [8].

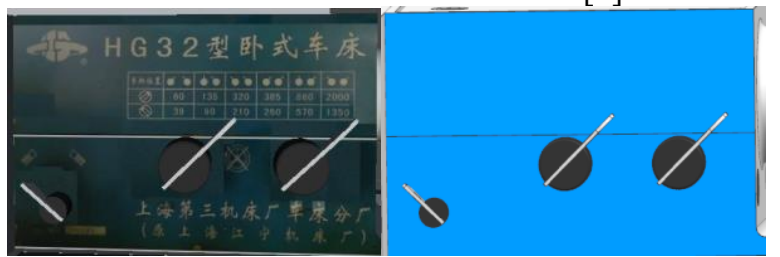

Figure 3. Comparison of the effect of map processing

\subsection{Development of factory roaming function}

Manual roaming is the most common virtual scene observation method. Through the upper and lower keys, the left and right movement of the model and the forward and reverse rotation are realized. The workshop roaming scene is shown in Figure 4. The typical program code is as follows:

if (Input.GetKey(KeyCode.A))

transform.Rotate $(0,20 *$ Time.deltaTime $*$ speed, 0 , Space.Self);

\}

In order to clearly observe the model of mechanical equipment in the workshop, the roaming system designed the scaling and rotation functions of the model by calling the ray function and the Lerp function. The function call statement is as follows:

ray=Camera.main.ScreenPointToRay(Input.mousePositio n);

transform.rotation $=$ Quaternion.Lerp(transform.rotation, mRotation, Time.deltaTime * Damping);

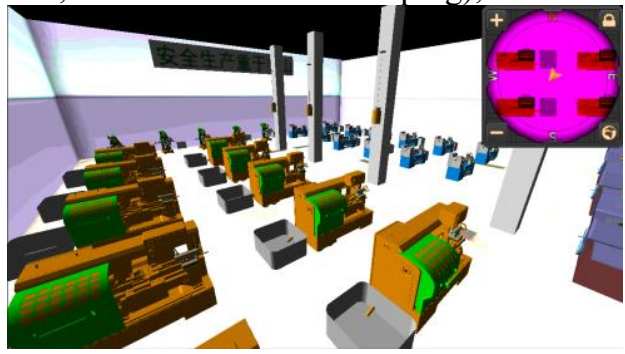

Figure 4. Workshop roaming scene

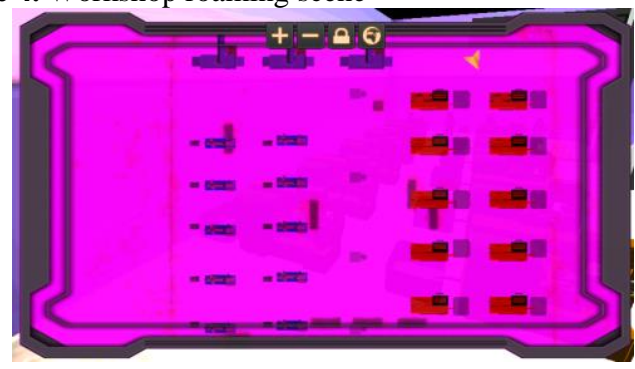

Figure 5. Small map technology

The workshop roaming usually requires the overall layout of the equipment. At this time the virtual camera cannot achieve the effect of the global observation. The system realizes the small map roaming technology in the workshop (Figure 5). The main functions are:

(1) It can display the orientation of the current scene in real time;

(2) It can reduce and put the size map to facilitate the rover to understand the current Location;

(3) Setting up map observation mode from two angles of facing and strabismus.

\subsection{Development of plant equipment layout function}

The rational design of the equipment layout is the key to the effective process of mechanical processing. The scientific and rational layout of equipment can improve the efficiency of the machining work, effectively save the production cost, ensure the quality and quantity of the mechanical processing, and also optimize the specific process of the production and processing.

Virtual factory design refers to the three-dimensional modeling of the workshop through the acquisition of physical workshops, production lines and logistics, and the building of a virtual manufacturing digital virtual workshop by using 3D design tools. Virtual factory layout has real-time and interactive layout function, and can be used for layout analysis in 3D scenario. Figure 6 is the free layout interface of the factory machine tool developed by the system. The mobile machine can automatically calculate the distance between the machine tool and the adjacent machine tools and display it in real 
time. Fig. 7 first select the scene for machine tool equipment. In the scene, not only the layout function is realized, but also the machine tool can be selected freely and the data is saved.

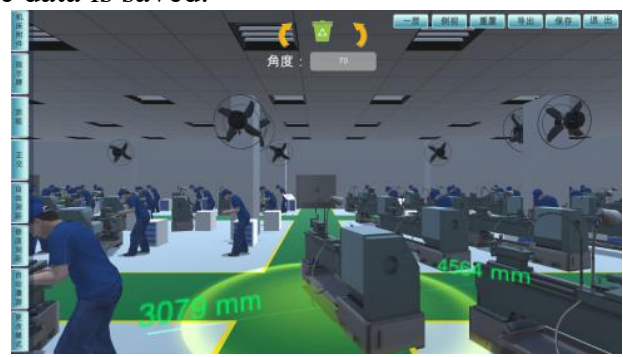

Figure 6. Machine tool layout scene

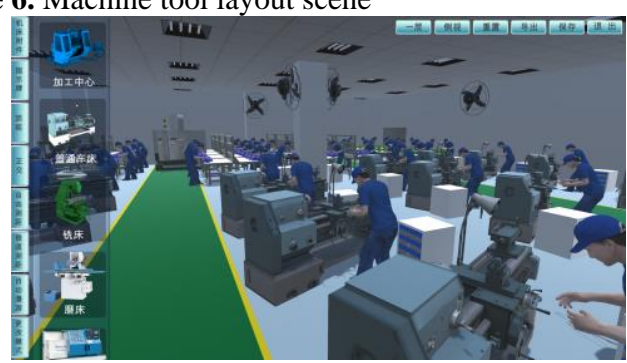

Figure 7. Machine tool selection scene

\subsection{Development of virtual lathe simulation function}

In the virtual simulation cutting scene, all the cutting technology and machine tool running data are collected from the actual cutting data, including the basic dimension parameters and the cutting parameters of the lathe. And the collected dynamic data are displayed in the processing scene by the way of mapping.

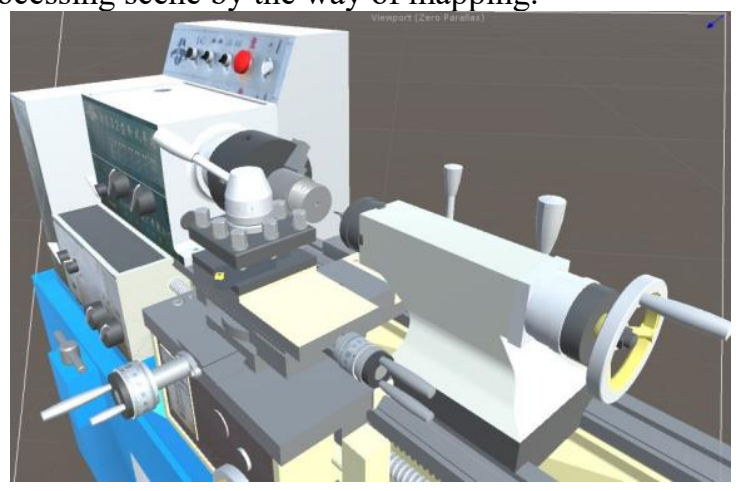

Figure 8. Simulation cutting scene

\section{Development of virtual workshop roaming VR system}

ZSpace system is a desktop virtual reality system that integrates real world work environment. The core technology is high fidelity stereo display system, low delay tracking system, stereo vision and direct interaction. Compared with the system developed by Unity3D, the virtual reality system developed by zSpace reflects the natural interactive and immersive of the new system.

\subsection{Basic principles of virtual reality}

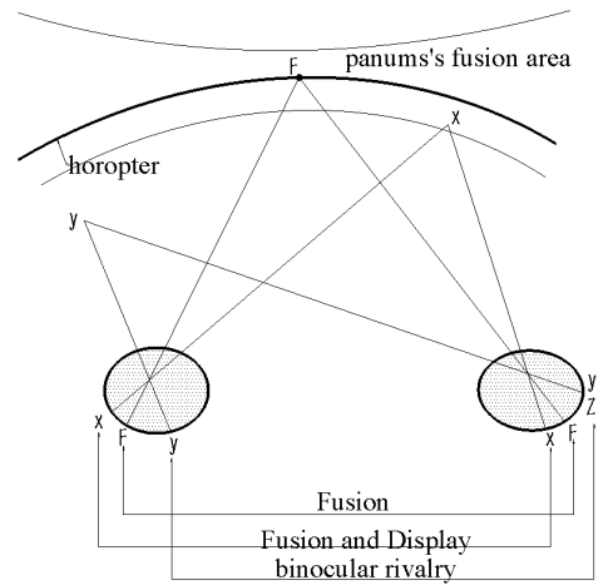

Figure 9. Stereoscopic imaging principle

Figure 3[9] describes the basic principle of zSpace stereoscopic display (Fig. 9). Assuming the fixed point $F$ from the top down view, through the fixed point binocular (Panum fusion area), there are object $\mathrm{X}$ and object $\mathrm{Y}$. When $\mathrm{F}$ is fixed, the $\mathrm{F}$ image stimulates the corresponding retinal points of two eyes and produces fusion. The object $\mathrm{X}$ is generated by cross parallax before binocular vision. Object $\mathrm{X}$ stimulates retinal differences in binocular fusion, as $\mathrm{X}$ falls within the fusion area of Panum. The object $Y$ is in the front of the two eye view, with a cross parallax. The retinal difference point of the image from the object $\mathrm{Y}$ stimulates two eyes as double, because $\mathrm{Y}$ is outside the Panum fusion zone. Because $\mathrm{Y}$ has two retinal images and is in very different retinal regions, the $\mathrm{Y}$ image in the left eye may stimulate the retina region corresponding to one area in the right eye area. This image comes from the $\mathrm{Z}$ images of different objects in the field of vision, which leads to the competition of the binocular and forms the stereoscopic display effect.

\subsection{Interactive design}

The traditional system interaction design is realized by calling the UI function, which is, clicking the button by mouse. This is slightly intelligent compared with the traditional multimedia courseware. But it cannot make the learners feel the sense of being in the situation from the degree of interaction and immersion. This section attempts to call some functions in zSpace to implement the interaction between objects and objects. Figure 10 interacts with the model and model to roam the first view of the workshop (the system is a stereoscopic interface, and the picture is photographed). 


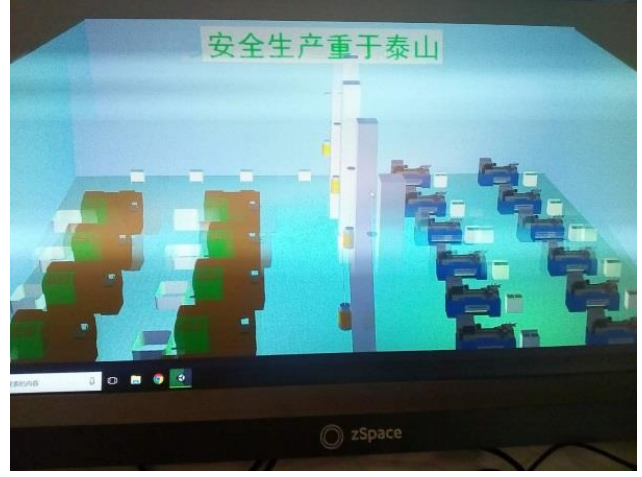

Figure 10. Total system interface

\subsection{Application of Augmented Reality Technology}

Augmented reality is a technology that combines real environment with virtual reality. Augmented reality system is usually designed for human vision, with graphic images as the main output media. Achieving more natural interaction between users and virtual objects in real scenes is one of the goals of augmented reality technology. Therefore, the display function and interaction function in augmented reality is the key to reflect the application value of the system.

This system has the function of virtual reality, and it also has the function of augmented reality. The zView device can share the stereoscopic model seen in the VR. It uses the high definition camera as the hardware, screen overlapping technology as software, to overlap the zSpace picture. The purpose of the technology is to screen the virtual world in the real world and interact with it. Fig. 11 is the augmented reality effect of the main structure of the lathe and gear in the virtual workshop.

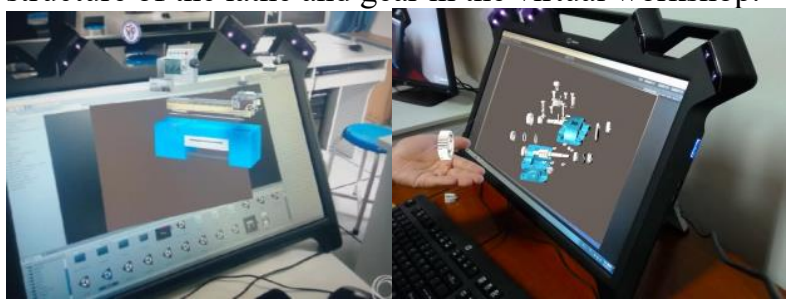

Figure 11. Augmented reality

\subsection{Conclusion and Prospect}

With the development of virtual reality technology, this technology has been applied in engineering and technology fields often and often. This system aims at developing virtual factories in the field of intelligent manufacturing. First, the system development framework is formulated, the function of the system is determined; the functions of virtual factory roaming and virtual equipment layout are developed. The system can quickly understand the layout of the plant equipment, as well as helping the factory managers to optimize the layout workshops reasonably. The production equipment has played an important role in saving time and improving efficiency. At the same time, in order to reflect the visual operation interface, the system becomes more interactive and immersive with the aid of virtual reality operation engine. The engine also provides some reference for the design of intelligent factory.

The follow-up study combines the virtual reality glove with the machine tool manufacturing process to develop a more advanced interpersonal interaction, which makes the virtual reality technology better serve the intelligent manufacturing process.

\section{Acknowledgements}

This work was supported by the Key Discipline Project of Shanghai Polytechnic University (No. XXKZD1605) and Graduate project fund of Shanghai Polytechnic University (No. A01GY17F022).

\section{References}

1. Shi Min, Wang Junzheng, Wei Jiahui. Realistic 3D virtual scene construction and roaming method [J]. Journal of System Simulation,2014,(9):1969$1974,1979$.

2. Zou Hong, Yang Hong, Cui Xiaowei, et al. Virtual simulation scene and interactive roaming system [J]. Oil-Gasfield Surface Engineering, 2014, (1): 22-23.

3. Hu Fuwen, Chen Chengkun, Liu Yancheng, Li Li. Research on virtual planning system for product assembly process $[\mathrm{J}]$. Mechanical design and manufacture, 2016, (12):180-183.

4. Yu Qiang, Li Xiangsong. Application Research of digital plant layout simulation technology [J]. Machinery \& Electronics, 2015, (11): 21-24.

5. Wen Huaixing, Huang Zhengxiang. Research on Virtual Assembly System Based on Virtools parallel machine tool [J]. Manufacturing Technology \& Machine Tools, 2017, (9): 91-94.

6. Liang Hongbao, Cheng Qi chao. Optimization of 3D model of virtual factory based on Virtools [J]. Computer and Digital Engineering, 2008,36 (11): 166.

7. Zhu Wenhua, Cai Bao, Shi Kun Ju, Liu Sai. Research on the disassembly and assembly of reducer combined with virtual and real [J]. Laboratory Research \& Exploration, 2017,36 (11): 92-108.

8. Hoai Nam Le Tran, Kabilen Sornum. A Systematic Approach for Rapid 3D Reconstruction from Photo Sets. 2010 11th Int. Conf. Control, Automation, Robotics and Vision Singapore. 2010(12): 11671174.

9. Patterson, R. Human factors of stereo displays: An update. Journal of the Society for Information Display, 2009(17): 987-996. 